\title{
Quantitative Comparison of Korotkoff Sound Waveform Characteristics: Effects of Static Cuff Pressures and Stethoscope Positions
}

\author{
Fan Pan ${ }^{1}$, Fei Chen ${ }^{2 *}$, Chen Liu ${ }^{3}$, Zhipeng Yang ${ }^{1}$, Zhihong Liu ${ }^{1}$, Dingchang Zheng ${ }^{4 *}$ \\ ${ }^{1}$ College of Electronic Engineering, Chengdu University of Information Technology, Chengdu \\ 610225, China \\ ${ }^{2}$ Department of Electrical and Electronic Engineering, Southern University of Science and \\ Technology, Shenzhen 518055, China \\ ${ }^{3}$ Sichuan Vocational and Technical College, Suining 629000, China \\ ${ }^{4}$ Department of Medical Science and Public Health, Faculty of Medical Science, Anglia \\ Ruskin University, Chelmsford, CM1 1SQ, UK
}

*Address correspondence to Fei Chen, Department of Electrical and Electronic Engineering, Southern University of Science and Technology, Shenzhen 518055, China. Electronic mail: fchen@sustc.edu.cn

*Address correspondence to Dingchang Zheng, Department of Medical Science and Public Health, Faculty of Medical Science, Anglia Ruskin University, Chelmsford, CM1 1SQ, UK. Electronic mail: dingchang.zheng@anglia.ac.uk 


\begin{abstract}
The underlying principles of Korotkoff sound (KorS) during blood pressure (BP) measurement and its waveform characteristic changes with cuff pressure and stethoscope position have not been fully understood. This study aimed to quantify the effects of cuff pressure and stethoscope position on the measured KorS waveform characteristics. Thirty healthy subjects were recruited in this study. Four stethoscopes were placed on the circumferential directions around the arm ( $\mathrm{m} 1, \mathrm{~m} 2, \mathrm{~m} 3$ and $\mathrm{m} 4 ; \mathrm{m} 1$ was above the artery, and equal distance between each other), and then sequentially at three different longitudinal positions ('upper', 'middle' and 'low' part under the cuff). At each longitudinal position, three levels of static cuff pressure (high: $\mathrm{SBP}+10 \mathrm{mmHg}$, low: DBP-10 $\mathrm{mmHg}$, and medium: $\mathrm{DBP}+(\mathrm{SBP}-\mathrm{DBP}) / 3$ ) were applied during the recording of KorS waveform. The averaged KorS waveform was firstly computed by using an interpolation method, separately for measurements from different stethoscope locations and cuff pressures. Two quantitative indices were derived to characterize the recorded KorS waveform: intensity amplitude and high-level duration of KorS waveform. Post-hoc pairwise comparisons after analysis of variance were used to compare the waveform characteristic differences between different stethoscope locations and between cuff pressures. Variance analysis demonstrated that the effects of stethoscope circumferential and longitudinal positions and cuff pressure on the two KorS waveform indices were significant (all $p<0.001$ ). In detail, KorS waveform recorded at cuff pressure $P_{\text {MEDIUM }}$ had larger intensity amplitude and shorter high-level duration than

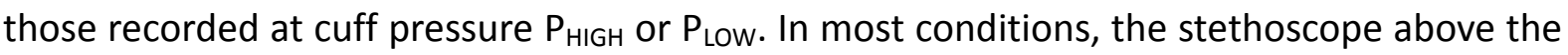
artery (m1) produced the largest RMS intensity amplitude and shortest high-level duration, while the stethoscope at the opposite location of $\mathrm{m} 1$ generated the smallest RMS intensity amplitude and longest high-level duration. In terms of the effect of longitudinal position, the stethoscopes below the middle of the cuff always produced KorS recordings with larger intensity amplitude and shorter high-level duration. This study has quantified and provided scientific evidence that cuff pressure, stethoscope longitudinal and circumferential positions are important factors influencing KorS waveform characteristics.
\end{abstract}

Keywords: Korotkoff sound, waveform characteristic, stethoscope position, cuff pressure. 


\section{INTRODUCTION}

Manual auscultatory blood pressure (BP) measurement is regarded as the gold standard of clinical BP measurement. ${ }^{1}$ The manual method is achieved by a trained clinician or nurse who uses the stethoscope to listen to the appearance and disappearance of Korotkoff sound (KorS). When the arm is under the atmospheric pressure without external cuff pressure, as the heart beats, the arterial pulses are transmitted smoothly through non-turbulent blood flow throughout the arteries, and no sound is produced. Regarding the biophysical mechanism of audible KorS during BP measurement, Korotkoff himself speculated that, with deflating cuff pressure, the appearance of KorS is associated with the sudden opening of the artery. ${ }^{2}$ As the pressure in the cuff drops further below the diastolic BP (DBP), the cuff no longer provides any restriction to blood flow allowing the blood flow to become smooth again with no turbulence and thus produce no further audible sound. But Babbs proposed that KorS is produced from the resonant motion of the arterial wall with deflating cuff pressure. ${ }^{3}$ Although the genesis of KorS is not fully understood, investigating the factors influencing KorS waveform features is without doubt physiologically and clinically important. ${ }^{4-7}$ Any potential small KorS feature changes and their associated factors worth further investigation, which would provide fundamental basis to improve BP measurement techniques and accuracy. ${ }^{8,9}$

It is generally known that KorS features change with deflating cuff pressure during BP measurement. Five distinct Korotkoff phases have been observed, which is described as following: phase I as clear tapping tone; phase II sounds become soften and have a swishing quality; phase III sounds like phase I but more sharpening; phase IV has a distinct abrupt muffling of sounds and phase $V$ represents the disappearance of the sounds. ${ }^{1,10,11}$ Rauerkus and Cozby investigated the frequency characteristics of the five different phases using Fourier transform methods, ${ }^{12,13}$ and Allen et al compared the difference of KorS features in association with different phases using a joint time-frequency analysis technique. ${ }^{14}$ Both investigations were conducted with deflating cuff pressure, and there is large human variation in identifying specific Korotkoff phases. To the best of our knowledge, there is no study to quantify the differences of KorS waveform characteristics under different static cuff pressures.

It is also known that the recorded KorS could be different with the stethoscope below the cuff at different positions on the arm. Our previous study has quantitatively demonstrated that the measured DBPs with the stethoscope at two different longitudinal positions (under and outside the cuff) were significantly different. ${ }^{5}$ However, our published study did not quantify the KorS waveform characteristic differences in association with different stethoscope positions, which would contribute towards a better understanding of how blood flow travels down the artery during BP measurement. Moreover, the effect on the KorS features of stethoscope position on circumferential direction around the arm has also not been comprehensively investigated.

The aim of this study was therefore to quantify and compare the difference in KorS waveform characteristics between simultaneous measurements of KorS at different static cuff pressures and at different longitudinal and circumferential positions under the BP measurement cuff. 


\section{MATERIALS AND METHODS}

\section{Subjects}

Thirty healthy subjects (20 male and 10 female) participated in this study. The experiment on human subjects received ethical permission from the Newcastle \& North Tyneside Research Ethics Committee (reference number: 11/NE/0340). The investigation conformed with the principles in the Declaration of Helsinki (1989) of the World Medical Association. All participants gave their written information consent before attending the experiments. Clinical information for all participants is summarized in Table 1. The data used for analysis were anonymous.

Table 1. General data information for the subjects studied.

\begin{tabular}{lcccc}
\hline \multicolumn{1}{c}{ Subject information } & & & & \\
\hline No. subjects & 30 & & & \\
No. male & 20 & & & \\
No. female & 10 & & & \\
& & & & \\
& Min & Max & Mean & SD \\
Age (years) & 23 & 56 & 33 & 9 \\
Height (cm) & 152 & 186 & 172 & 8 \\
Weight (kg) & 47 & 90 & 71 & 14 \\
Arm circumference (cm) & 20 & 35 & 26 & 3 \\
Systolic blood pressure $(\mathrm{mmHg})$ & 83 & 140 & 110 & 11 \\
Diastolic blood pressure $(\mathrm{mmHg})$ & 58 & 85 & 70 & 6 \\
Mean arterial pressure $(\mathrm{mmHg})$ & 70 & 96 & 82 & 6 \\
\hline
\end{tabular}

\section{Data Acquisition}

All data acquisitions were performed in a quiet and temperature-controlled clinical measurement room by one trained operator. Before acquisition the subject was asked to rest on a chair for 5 minutes. During the acquisition, subjects were also asked to breathe gently.

As shown in Figure 1 (a), four identical stethoscopes were placed on the circumferential direction around the arm. They were located at 0, 90, 180 and 270 azimuth angles (noted as $\mathrm{m} 1, \mathrm{~m} 2, \mathrm{~m} 3$ and $\mathrm{m} 4$, respectively), and $\mathrm{m} 1$ at 0 azimuth was placed above the artery. As illustrated in Figure 1 (b), stethoscopes were then sequentially placed under the cuff at three longitudinal positions (upper, middle and low). At each longitudinal position, three different levels of cuff pressure $\left(\mathrm{P}_{\text {HIGH }}=\mathrm{SBP}+10 \mathrm{mmHg}, \mathrm{P}_{\text {MEDIUM }}=\mathrm{DBP}+(\mathrm{SBP}-\mathrm{DBP}) / 3 \mathrm{mmHg}\right.$ and $\mathrm{P}_{\text {LOW }}=$ DBP-10 $\mathrm{mmHg}$ ) were applied and maintained at a static level in the cuff during the KorS recording. 


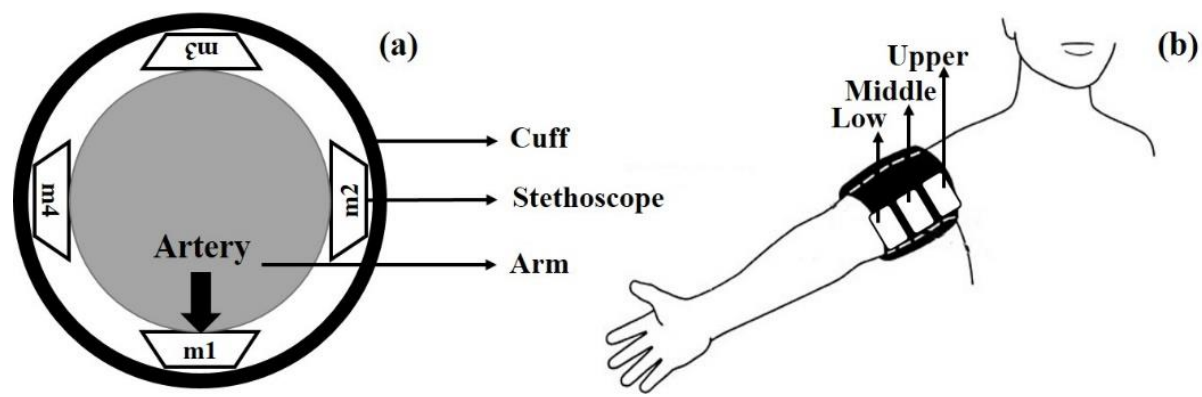

Fig 1. Illustration of the circumferential and longitudinal positions of stethoscopes under the cuff

For each subject, there were 2 repeated sessions with 9 recordings, giving a total of 18 recordings. For each recording, four channels of KorS signal from the four identical stethoscopes, one channel of ECG signal and one channel of cuff pressure signal were simultaneously and digitally recorded for 45 seconds by a bespoke recording system. The system for recording KorS signal mainly included a cardiology stethoscope (A type, Yuwell Medical Equipment \& Supply Co., Ltd. China), a microphone (WM-60A, Panasonic Co., Ltd.), an audio amplifier, and a data capture computer. The bandwidth of the recording system for the KorS signal was between $20 \mathrm{~Hz}$ to $800 \mathrm{~Hz}$ (anti-aliasing filter cut-off frequency). The computer digitized the signals at a sampling rate of $2000 \mathrm{~Hz}$ using an analogue-to-digital convertor with 16 bits resolution (National Instruments, DAQ-516 card).

For the 9 recordings within a session, 3 different static cuff pressures and 3 different stethoscopes longitudinal positions were applied sequentially, and the sequence of these different levels of static cuff pressure and stethoscope longitudinal positions were randomised between subjects. A time interval of at least 5 minutes was given between sessions, and at least 1 minute between the 9 recordings within a session, allowing full recovery of cardiovascular hemodynamics after the release of cuff pressure.

\section{KorS Waveform Averaging}

Considering the relatively high-quality of ECG waveform recording, the ECG R-wave peaks were firstly located, and used as a reference for beat-to-beat segmentation of KorS. Figure 2 illustrates the beat-to-beat segmentation of KorS. The dashed lines in Figure 2 marking the locations of ECG R-wave peaks, split the KorS waveform into multiple segments. Due to the heart rate variance, the duration of each KorS waveform varies, making the KorS segments have different durations; Hence, a linear interpolation processing was implemented to ensure each KorS waveform was re-sampled with equal number of points. The target number of points was chosen corresponding to the shortest KorS segment. All those processed KorS waveforms with the same number of points were averaged, rending a noise-suppressed and averaged KorS waveform. Note that the mean of each interpolated KorS waveform was adjusted to the mean of its un-interpolated KorS waveform. Hence, the interpolation process did not change the mean of KorS waveform. Figure 3 gives some examples of averaged KorS waveforms recorded with different cuff pressures and at different stethoscope longitudinal positions, and Figure 4 shows their corresponding spectra. Figure 5 plots the averaged KorS waveforms at the four circumferential positions around the arm. 


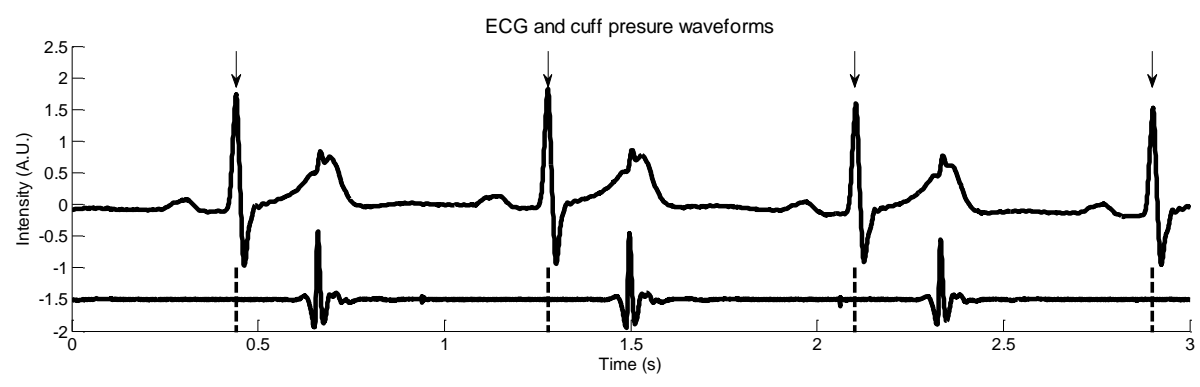

Fig 2. Illustration of beat-to-beat segmentation of ECG and KorS waveforms. The arrows and dashed lines are marked as the locations of ECG R-wave peaks.
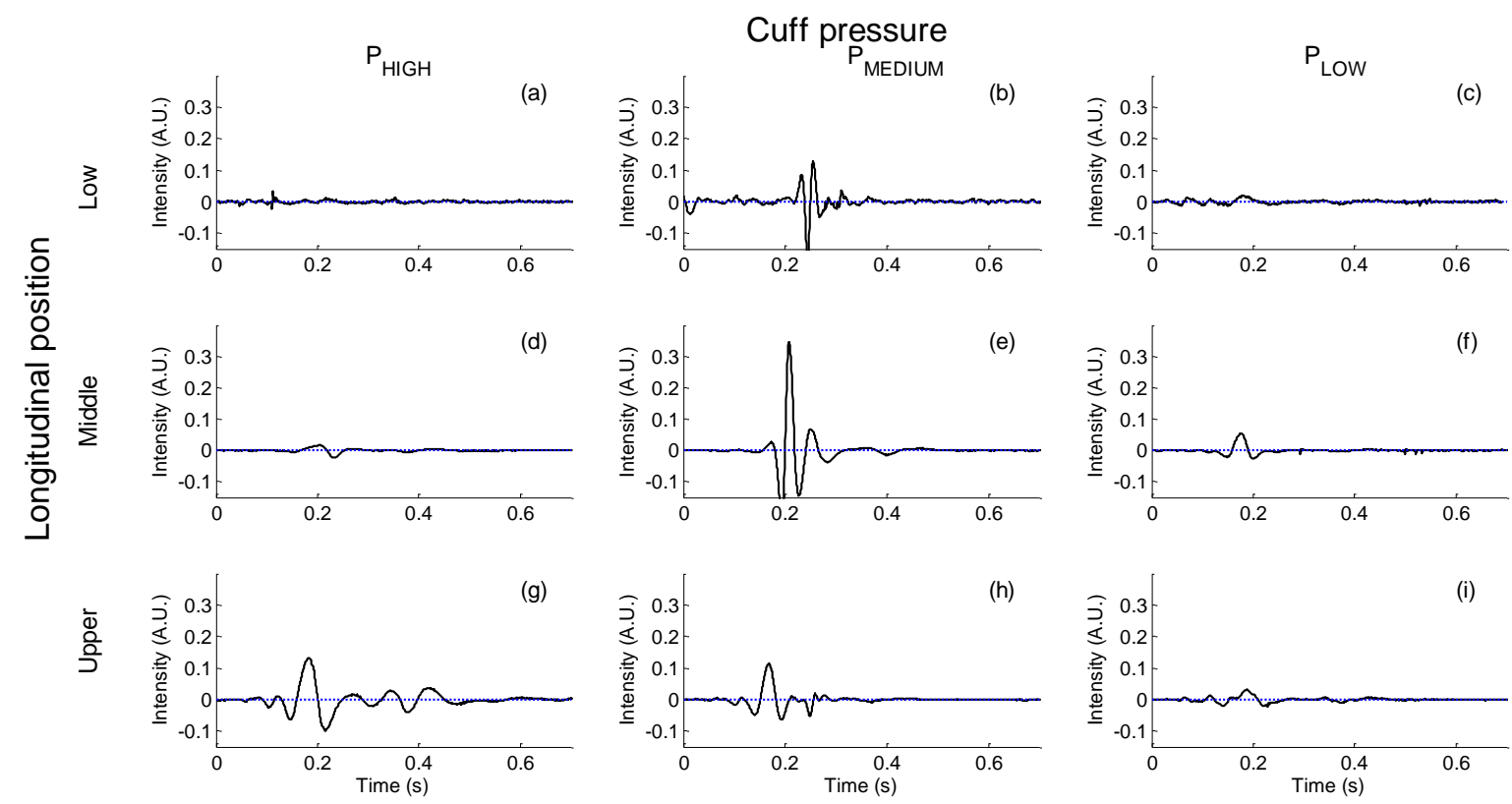

Fig 3. Examples of averaged KorS waveforms measured at three longitudinal positions (upper, middle and low $)$ and three levels of cuff pressure $\left(\mathrm{P}_{\text {HIGH }}=\mathrm{SBP}+10 \mathrm{mmHg}, \mathrm{P}_{\text {MEDIUM }}=\right.$ $\mathrm{DBP}+(\mathrm{SBP}-\mathrm{DBP}) / 3 \mathrm{mmHg}$ and $\left.\mathrm{P}_{\mathrm{LOW}}=\mathrm{DBP}-10 \mathrm{mmHg}\right)$. 


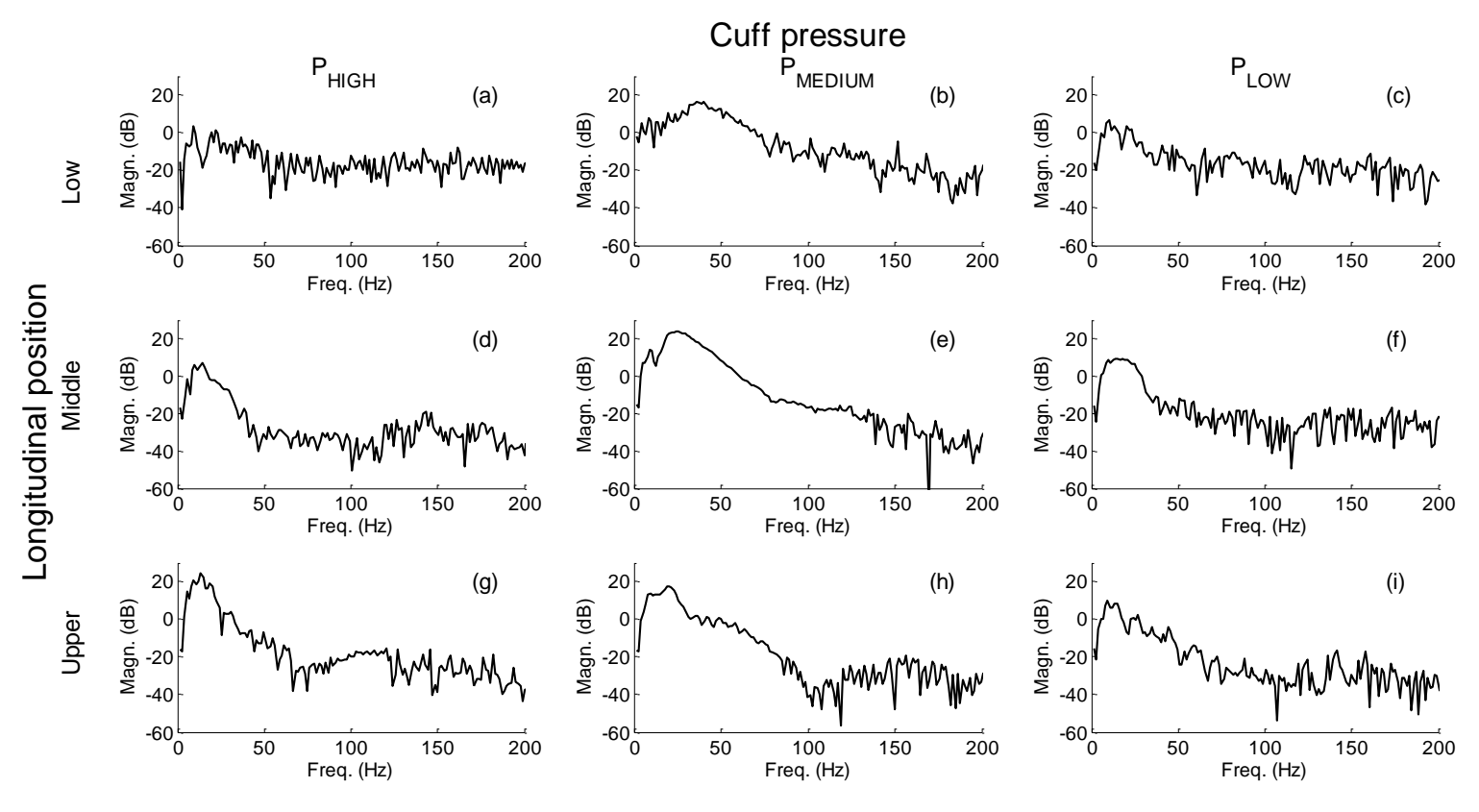

Fig 4. The spectra of averaged KorS waveforms measured at three longitudinal positions (upper, middle and low) and three levels of cuff pressure $\left(P_{H I G H}, P_{\text {MEDIUM }}\right.$ and $P_{\text {LOW }}=$ DBP-10 $\mathrm{mmHg}$ ). The spectrum is corresponding to the temporal waveforms shown in Figure 3.
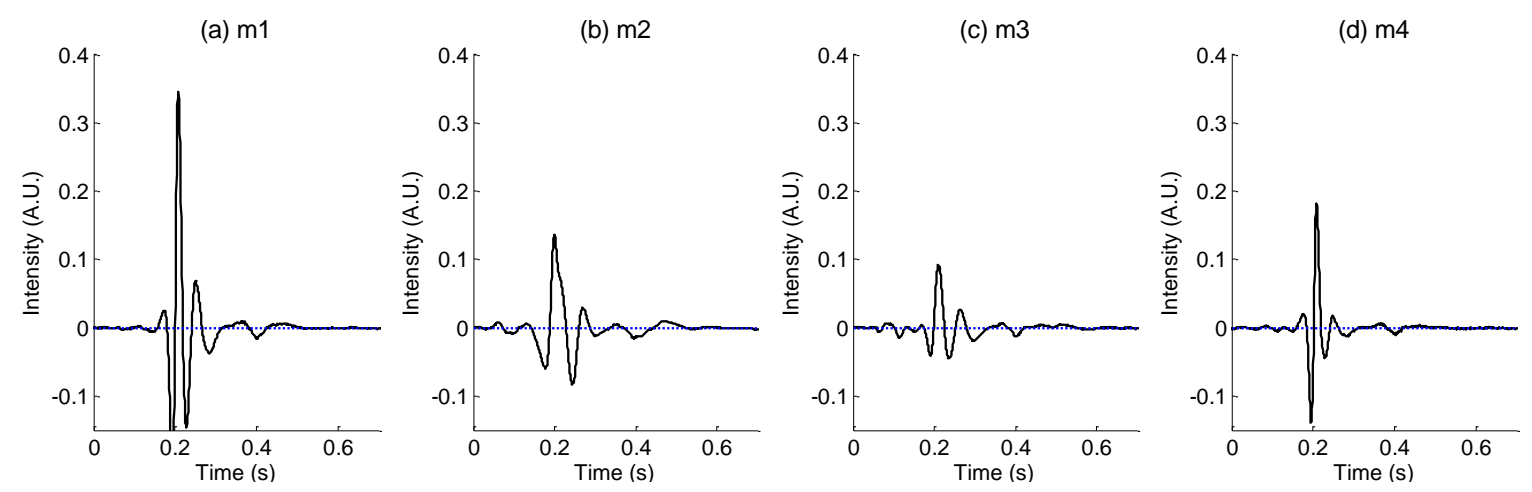

Fig 5. Examples of averaged KorS waveforms measured at four circumferential stethoscope positions ( $m 1, m 2, m 3$, and $m 4)$. Note: in this example, the stethoscope was at the middle longitudinal position, and the cuff pressure was $\mathrm{P}_{\text {MEDIUM. }}$

\section{RMS Intensity Amplitude of KorS Waveform}

Intensity amplitude of KorS waveform was derived as the first parameter. The root mean square (RMS) level of short-term segment (16 ms in this study) was computed, and the amplitude of the RMS intensity profile was defined as the RMS intensity amplitude of KorS waveform. Figure 6 (b) and (e) show an example of RMS intensity profile (and its amplitude) of a KorS waveform. 

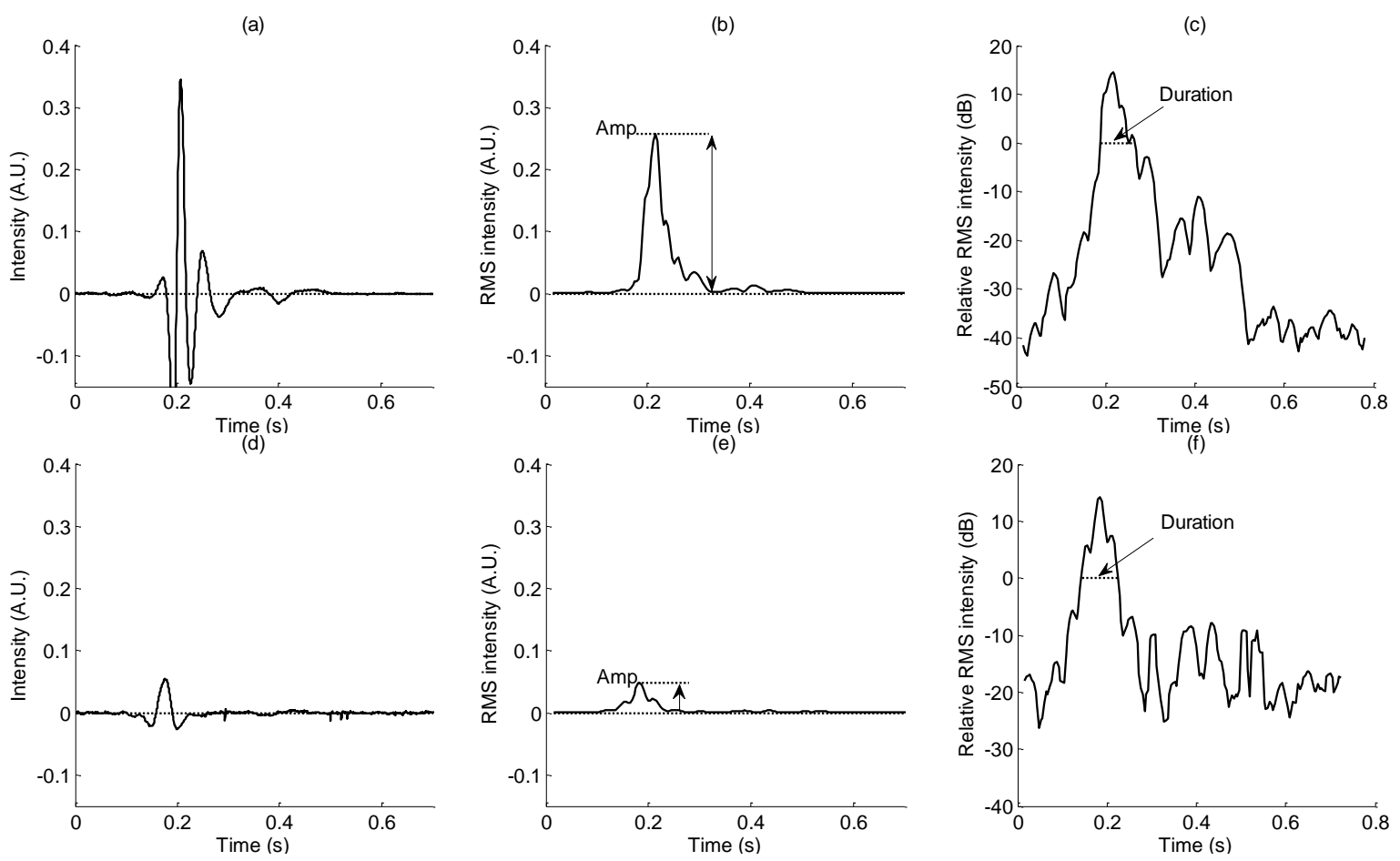

Fig 6. Illustrations of two KorS waveforms in (a) and (d), their RMS intensity amplitude in (b) and (e), and high-level durations of RMS intensities in (c) and (f).

\section{High-level Duration of KorS Waveform}

The RMS-level based segmentation has been commonly used in a number of acoustic signal processing to characterize the duration of waveform of interest. ${ }^{15,16}$ The high-level duration of KorS (in response to the overall RMS value of KorS waveform) was used to characterize the time-spanning property of KorS waveform, which was computed as follows: The KorS waveform was first divided into short-term (16 ms in this study) segments. The RMS value of each segment was computed and compared with that of the whole KorS waveform. Those segments with RMS values larger than that of the whole KorS waveform [i.e., relative RMS intensity above $0 \mathrm{~dB}$ in Figure 6 (c) and (f)] were identified into high-level segment. Figure 6 (c) and (f) in logarithmic scale exemplified the high-level segment of a KorS waveform. The duration of those points above the dashed line (i.e., relative RMS intensity of $0 \mathrm{~dB}$ ) was defined as the high-level duration.

\section{Statistical Analysis}

Statistical significance was determined by using the RMS intensity amplitude or high-level duration of KorS waveform as the dependent variable, and cuff pressure, stethoscope circumferential position and longitudinal position as the three within-subject factors. Post-hoc pairwise comparisons, according to Fisher's Least Significant Difference (LSD) test, were performed using SPSS to compare the intensity amplitude or high-level duration of KorS waveform between different cuff pressures, stethoscope circumferential positions and longitudinal positions. A $p<0.05$ was considered to be statistically significant.

\section{RESULTS}




\section{RMS Intensity Amplitude of KorS Waveform}

First, when comparing the RMS intensity amplitude of KorS waveform between the two sessions, paired t-test showed that there was no significant difference $(p>0.05)$ at the same cuff pressure, stethoscope circumferential position and longitudinal position. Statistical significance was determined by using the RMS intensity amplitude (averaged across two sessions) of KorS waveform as the dependent variable, and cuff pressure, stethoscope circumferential position and longitudinal position as the three within-subject factors. Three-way analysis of variance (ANOVA) with repeated measurements indicated a significant effect on RMS intensity amplitude of stethoscope circumferential position $(F[3,87]=62.80$, $p<0.001)$, stethoscope longitudinal position $(F[2,58]=14.25, p<0.001)$ and cuff pressure $(F[2,58]=49.40, p<0.001)$. It is noted that when taking all intensity amplitude into consideration, those measured at cuff pressure $P_{\text {MEDIUM }}$ were larger than those at $P_{\text {HIGH }}$ or Plow.

Figure 7 shows the measurement of RMS intensity amplitude (averaged across two sessions) of KorS waveform across all conditions. In detail, as noted by sign ' + ' in Figure 7 (a) and (b), when KorS waveform was measured at stethoscope longitudinal position 'upper' and cuff pressure $\mathrm{P}_{\text {HIGH }}$, and at longitudinal position 'middle' and cuff pressure $\mathrm{P}_{\text {MEDIUM, }}$, the RMS intensity amplitude from stethoscope circumferential position $\mathrm{m} 1$ was significantly larger than those from position $\mathrm{m} 2-\mathrm{m} 4$ (all $p<0.01$ ). However, with cuff pressure $\mathrm{P}_{\text {Low, }}$ as shown in Figure 7 (c), at stethoscope longitudinal position 'low' or 'upper', the intensity amplitude from $\mathrm{m} 2$ was significantly larger than those from $\mathrm{m} 1, \mathrm{~m} 3$ and $\mathrm{m} 4$, which differed from the advantage of high amplitude at stethoscope circumferential position $\mathrm{m} 1$ observed from Figure 7 (a) and (b).

Paired comparisons also demonstrated that, as shown in Figure 7 (a), with cuff pressure at $\mathrm{P}_{\text {HIGH, }}$ RMS intensity amplitude measured at stethoscope longitudinal position 'upper' was significantly larger than those at longitudinal positon 'middle' or 'low'. With cuff pressure at $\mathrm{P}_{\text {MEDIUM }}$ or $\mathrm{P}_{\text {Low, }}$ the highest RMS intensity amplitude was measured at stethoscope longitudinal position 'middle', which significantly larger than those measure from 'upper' or 'low', as noted by asterisk signs ' ${ }^{* \prime}$ in Figure 7 (b) and (c). 
(a) $P_{\text {HIGH }}$

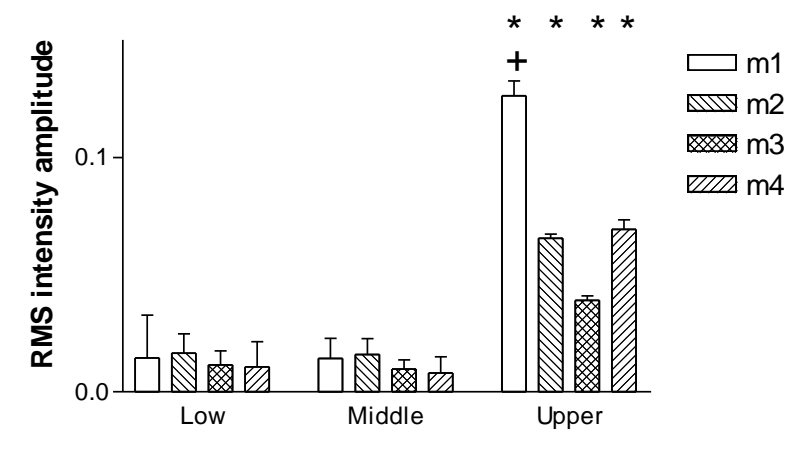

(b) $P_{\text {MEDIUM }}$

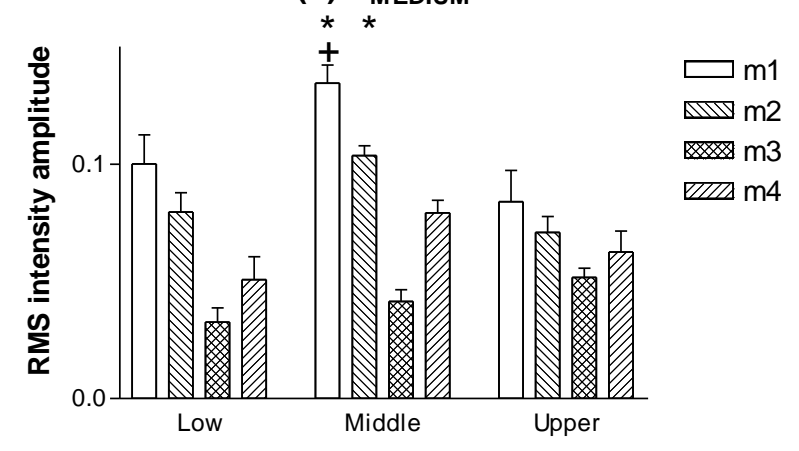

(c) PLow

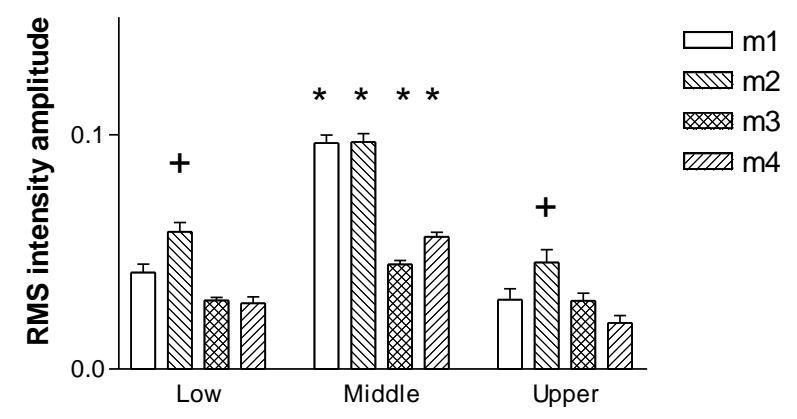

Fig 7. RMS intensity amplitude of KorS waveform across all conditions (thee cuff pressures: $\mathrm{P}_{\text {HIGH }}, \mathrm{P}_{\text {MEDIUM }}$ and $\mathrm{P}_{\text {Low }}$; four circumferential positions: $\mathrm{m} 1, \mathrm{~m} 2, \mathrm{~m} 3$ and $\mathrm{m} 4$; and three longitudinal positions: low, middle and upper). Plus sign ' + ' denotes that the RMS intensity amplitude value was significantly $(p<0.01)$ larger than the other three values from different circumferential positions measured at the same stethoscope longitudinal position and cuff pressure. Asterisk sign ' ${ }^{* \prime}$ denotes that the amplitude value was significantly $(p<0.02)$ larger than the other two values from different longitudinal positions measured at the same stethoscope circumferential position and cuff pressure.

\section{High-level Duration of KorS Waveform}

First, when comparing the high-level duration of KorS waveform between the two sessions, paired t-test showed that there was no significant difference $(p>0.05)$ at the same cuff pressure, stethoscope circumferential position and longitudinal position. Statistical significance was determined by using the high-level duration (averaged across two sessions) of KorS waveform as the dependent variable, and cuff pressure, stethoscope circumferential 
position and longitudinal position as the three within-subject factors. Three-way ANOVA with repeated measurements indicated a significant effect on high-level duration of KorS of stethoscope circumferential position $(F[3,87]=33.74, p<0.001)$, stethoscope longitudinal position $(F[2,58]=18.84, p<0.001)$ and cuff pressure $(F[2,58]=92.36, p<0.001)$. Noted that when taking all conditions into consideration, most high-level durations with cuff pressure $\mathrm{P}_{\text {MEDIUM }}$ were shorter than those measured at cuff pressure $\mathrm{P}_{\text {HIGH }}$ or $\mathrm{P}_{\text {Low }}$ in Figure 8.

Figure 8 shows the values of high-level duration (averaged across two sessions) of KorS waveform across all conditions. When the cuff pressure was $\mathrm{P}_{H I G H}$, KorS waveforms measured at stethoscope longitudinal position 'upper' produced shorter high-level duration compared to those from longitudinal position 'low' or 'middle', as noted by asterisk signs '*' in Figure 8 (a). However, when the cuff pressure was $P_{\text {MEDIUM }}$ or PLow, the stethoscope at longitudinal position 'middle' tended to generate KorS waveforms with shorter high-level duration.

(a) $P_{\text {HIGH }}$

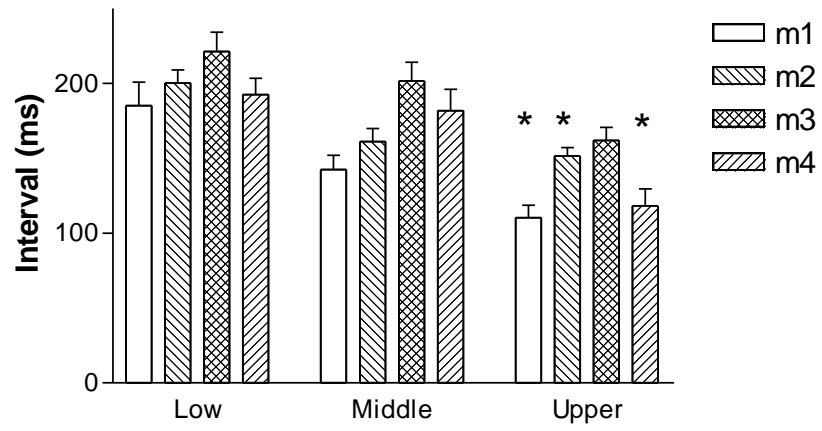

(b) $\mathbf{P}_{\text {Medium }}$

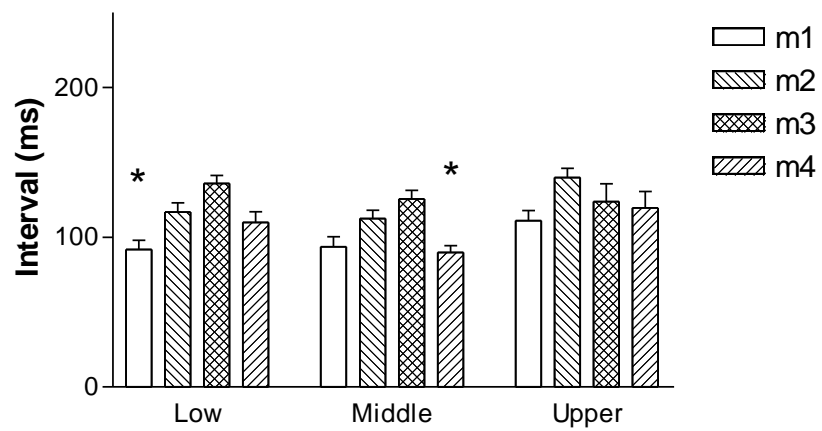

(c) $P_{\text {LoW }}$

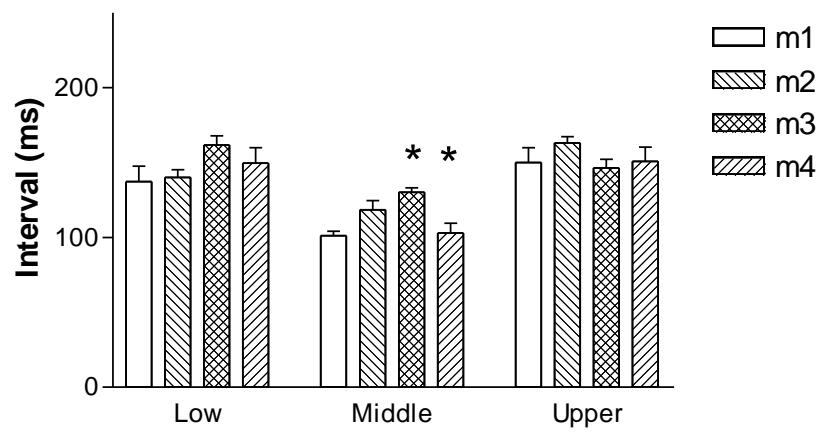


Fig. 8 High-level duration of KorS waveform across all conditions (thee cuff pressures: $P_{H I G H}$, $\mathrm{P}_{\text {MEDIUM }}$ and $\mathrm{P}_{\text {Low }}$; four circumferential positions: $\mathrm{m} 1, \mathrm{~m} 2, \mathrm{~m} 3$ and $\mathrm{m} 4$; and three longitudinal positions: low, middle and upper). Asterisk sign ${ }^{\prime * \prime}$ denotes that the interval value was significantly $(p<0.02)$ shorter than the other two values from different longitudinal positions measured at the same stethoscope circumferential position and cuff pressure.

Table 2 shows the results from the post-hoc multiple comparisons. In most conditions with the cuff pressures of $\mathrm{P}_{\text {HIGH }}$ and $\mathrm{P}_{\text {MEDIUM, }}$ the duration measured at position $\mathrm{m} 1$ (above the artery) was significantly shorted than that measured at position $\mathrm{m} 3$ (i.e. most away from artery), suggesting the importance of placing stethoscope close to the artery. This significantly shorter high-level duration was also observed between $\mathrm{m} 1$ and $\mathrm{m} 2$ with cuff pressure $\mathrm{P}_{\text {MEDIUM }}$ (at all three longitudinal cuff positions). However, the high-level duration differences between stethoscope longitudinal positions $\mathrm{m} 1$ and $\mathrm{m} 4$ were not significant in most conditions.

Table 2. Paired comparison of high-level durations of KorS waveforms. ' $s$ ' denotes that there was significant difference of high-level duration of KorS waveform between the two comparison at different circumferential position.

\begin{tabular}{|c|c|c|c|c|}
\hline \multirow{2}{*}{ Cuff pressure } & \multirow{2}{*}{$\begin{array}{c}\text { Stethoscope } \\
\text { longitudinal } \\
\text { position }\end{array}$} & \multicolumn{3}{|c|}{ Paired stethoscope circumferential position } \\
\hline & & $\mathrm{m} 1$ vs. $\mathrm{m} 2$ & $\mathrm{~m} 1$ vs. $\mathrm{m} 3$ & $\mathrm{~m} 1$ vs. $\mathrm{m} 4$ \\
\hline \multirow{3}{*}{$\mathrm{P}_{\mathrm{HIGH}}$} & Low & & $\mathrm{s}$ & \\
\hline & Middle & & $\mathrm{s}$ & $\mathrm{s}$ \\
\hline & Upper & $\mathrm{s}$ & $\mathrm{s}$ & \\
\hline \multirow{3}{*}{$\mathrm{P}_{\text {MEDIUM }}$} & Low & $\mathrm{s}$ & $\mathrm{s}$ & \\
\hline & Middle & $\mathrm{s}$ & $\mathrm{s}$ & \\
\hline & Upper & $\mathrm{s}$ & & \\
\hline \multirow{3}{*}{$P_{\text {Low }}$} & Low & & & \\
\hline & Middle & $\mathrm{s}$ & $\mathrm{s}$ & \\
\hline & Upper & & & \\
\hline
\end{tabular}

\section{DISCUSSION}

This study has quantified the effects of cuff pressure, stethoscope longitudinal and circumferential positions on the KorS waveform characteristics. To the best of our knowledge, this is the first experimental study to compare the KorS waveform characteristics among four circumferential positions, three longitudinal positions and three static cuff pressures.

The first finding is that, when the cuff pressure was above SBP, KorS can only be recorded from the stethoscope placed at the upper position under the cuff. The RMS intensity amplitude and high-level duration at the upper position are statistically more significant than those at the middle and low positions. This suggests that, even when artery is closed by external high cuff pressure, the upper edge of cuff still can be impacted by blood flow. In addition, when the cuff pressure was at MAP or below DBP, the KorS waveform recorded at the middle position had larger intensity amplitude and shorter high-level duration than those at the upper and low positions. This shows that, because of the edge effect, ${ }^{17}$ the artery in the middle of the cuff stresses significantly even the blood flow passes smoothly at low cuff pressure, 
This study also demonstrated that KorS waveform recorded at cuff pressure $\mathrm{P}_{\text {MEDIUM }}$ had larger intensity amplitude and shorter high-level duration than those recorded at cuff pressure $P_{\text {HIGH }}$ or $P_{\text {Low. }}$. Drzewiecki's study showed that the maximum amplitude of blood volume pulse occurs when the arterial compliance is maximum (when the transmural pressure is zero), while the zero transmural pressure happens when external cuff pressure equals to the MAP. ${ }^{18}$ In this study PMEDIUM was the same as the MAP; therefore, this study provided quantitative evidence that the genesis of KorS waveform had similar mechanism with blood volume pulse.

In addition, Figure 4 shows that cuff pressure may affect the spectral features of KorS waveforms. Taking the three spectra in Figure 4 (g), (h) and (i) (whereas the stethoscope was placed at the upper position under the cuff) as an example, it is seen that the frequency bandwidth of the KorS waveform recorded at cuff pressure $\mathrm{P}_{\text {MEDIUM }}$ covers a wider range than those recorded at cuff pressures $\mathrm{P}_{\text {HIGH }}$ and $\mathrm{P}_{\text {Low. }}$. The same findings are also seen for the stethoscope positions Middle and Low in Figure 4. This indicates that KorS waveform recorded at cuff pressure $P_{\text {MEDIUM }}$ has less attenuation to high frequency components than those recorded at cuff pressure $\mathrm{P}_{\mathrm{HIGH}}$ or $\mathrm{P}_{\text {Low. }}$.

Another significant finding of this study is that, in most conditions, stethoscope circumferential position $\mathrm{m} 1$ produced the largest RMS intensity amplitude and shortest high-level duration, while the stethoscope at the opposite of the $\mathrm{m} 1$ generated the smallest RMS intensity amplitude and longest high-level duration. This could be partially because KorS is originated from the artery. ${ }^{2,3}$ So, the closer to where the artery is, the stronger KorS waveform can be recorded. Our study provided scientific evidence for the guideline of manual BP measurement, where it recommends that the stethoscope should be placed gently over the brachial artery. ${ }^{1,19}$

The final key finding is that, in most conditions, the stethoscope placed below the middle of the cuff produced better KorS signals with larger RMS intensity amplitude and shorter high-level duration. In clinical practice of manual auscultatory BP measurement, stethoscope is commonly placed under the cuff close to the lower edge (i.e. the low position under the cuff in this study), or outside the cuff over the brachial artery in the antecubital fossa. ${ }^{1,20}$ Our previous study provided quantitative evidence and confirmed that manual DBP measurement from the stethoscope under the cuff could achieve closer values to the true invasive measurement in comparison with the stethoscope outside the cuff, because the KorS from the stethoscope under the cuff could be heard more easily. ${ }^{5}$ Based on the KorS waveform characteristic of the current study, it can be inferred that placing the stethoscope under the middle of the cuff over the brachial artery could be another option of better BP measurement, although further clinical investigations on the effect of stethoscope positions during BP reading is required. In addition, some automated devices incorporate a microphone in the cuff and use an electronic auscultatory method for BP measurement. In this study, the microphones were placed under the cuff with direct skin contact. As a future study, it would be therefore interesting to investigate the waveform characteristic difference between the microphone in cuff and under the cuff.

In summary, the effects of cuff pressure, stethoscope longitudinal and circumferential positions have been quantified in this work, providing scientific evidence that they are important factors influencing KorS waveform characteristics.

\section{ACKNOWLEDGEMENTS}


The experiment was conducted with the support from the Engineering and Physical Sciences Research Council (EPSRC) Healthcare Partnership Award (reference number EP/I027270/1). Fan Pan was sponsored by a visiting scholarship to study at Newcastle University between 2012 and 2013.

\section{REFERENCES}

1. Beevers G., G. Y. Lip and E. O'Brien. ABC of hypertension: Blood pressure measurement. Part II-conventional sphygmomanometry: technique of auscultatory blood pressure measurement. BMJ 322: 1043-1047, 2001.

2. Chungcharoen D. Genesis of Korotkoff Sounds. Am J Physiol 207: 190-194, 1964.

3. Babbs C. F. The origin of Korotkoff sounds and the accuracy of auscultatory blood pressure measurements. J Am Soc Hypertens 9: 935-950 e933, 2015.

4. Drzewiecki G. M., J. Melbin and A. Noordergraaf. The Korotkoff sound. Ann Biomed Eng 17: 325-359, 1989.

5. Pan F., D. C. Zheng, P. Y. He and A. Murray. Does the position or contact pressure of the stethoscope make any difference to clinical blood pressure measurements an observational study. Medicine 93: 2014.

6. Nunez A., M. Ihsan, S. Gaballah, A. Haque, O. Yurevich, S. Ahluwalia, L. Salciccioli, M. Stewart and J. Lazar. Differential effects of hyperemia and passive leg raising on QRS to Korotkoff sound time intervals in hypertensive patients. Journal of the American Society of Hypertension 10: e32, 2016.

7. Liu C., C. Griffiths, A. Murray and D. Zheng. Comparison of stethoscope bell and diaphragm, and of stethoscope tube length, for clinical blood pressure measurement. Blood Press Monit 21: 178-183, 2016.

8. Handler J. The importance of accurate blood pressure measurement. Perm J 13: 51-54, 2009.

9. Zheng D., L. Y. Di Marco and A. Murray. Effect of respiration on Korotkoff sounds and oscillometric cuff pressure pulses during blood pressure measurement. Med Biol Eng Comput 52: 467-473, 2014.

10. Geddes L. A. Handbook of blood pressure measurement. Clifton, N.J.: Humana Press, 1991, p. viii, 168 p.

11. Geddes L. A., H. E. Hoff and A. S. Badger. Introduction of the auscultatory method of measuring blood pressure--including a translation of Korotkoff's original paper. Cardiovasc Res Cent Bull 5: 57-74, 1966.

12. Rauterkus T., J. F. Feltz and J. W. Fickes. Frequency analysis of Korotkov blood pressure sounds using the Fourier transform. Sam-TR-66-8. Technical report SAM-TR. USAF School of Aerospace Medicine 1-46, 1966.

13. Cozby R. C. and R. R. Adhami. Low-frequency Korotkoff signal analysis and application. IEEE Trans Biomed Eng 40: 1067-1070, 1993.

14. Allen J., T. Gehrke, J. J. O'Sullivan, S. T. King and A. Murray. Characterization of the Korotkoff sounds using joint time-frequency analysis. Physiol Meas 25: 107-117, 2004.

15. Kates J. M. and K. H. Arehart. Coherence and the speech intelligibility index. Journal of the Acoustical Society of America 117: 2224-2237, 2005.

16. Chen F. and P. C. Loizou. Contributions of cochlea-scaled entropy and consonant-vowel boundaries to prediction of speech intelligibility in noise. Journal of the Acoustical Society of America 131: 4104-4113, 2012. 
17. Ku D. N. Blood flow in arteries. Annual Review of Fluid Mechanics 29: 399-434, 1997.

18. Drzewiecki G., R. Hood and H. Apple. Theory of the oscillometric maximum and the systolic and diastolic detection ratios. Ann Biomed Eng 22: 88-96, 1994.

19. O'Brien E., R. Asmar, L. Beilin, Y. Imai, J. M. Mallion, G. Mancia, T. Mengden, M. Myers, P. Padfield, P. Palatini, G. Parati, T. Pickering, J. Redon, J. Staessen, G. Stergiou, P. Verdecchia and M. European Society of Hypertension Working Group on Blood Pressure. European Society of Hypertension recommendations for conventional, ambulatory and home blood pressure measurement. J Hypertens 21: 821-848, 2003.

20. Pickering T. G., J. E. Hall, L. J. Appel, B. E. Falkner, J. Graves, M. N. Hill, D. W. Jones, T. Kurtz, S. G. Sheps, E. J. Roccella, P. Subcommittee of and R. Public Education of the American Heart Association Council on High Blood Pressure. Recommendations for blood pressure measurement in humans and experimental animals: Part 1: blood pressure measurement in humans: a statement for professionals from the Subcommittee of Professional and Public Education of the American Heart Association Council on High Blood Pressure Research. Hypertension 45: 142-161, 2005. 